\title{
Adjuvant Chemotherapy for Stage II Colon Cancer: Everyone Still Needs a Tailor
}

\author{
Michael F. McGee, MD, FACS ${ }^{1}$ and Al B. Benson III, MD, FACP, FASCO ${ }^{2}$ \\ ${ }^{1}$ Division of Gastrointestinal and Oncologic Surgery, Department of Surgery, Robert H. Lurie Comprehensive Cancer \\ Center of Northwestern University, Feinberg School of Medicine, Chicago, IL; ${ }^{2}$ Division of Hematology/Oncology, \\ Department of Medicine, Robert H. Lurie Comprehensive Cancer Center of Northwestern University, Feinberg School of \\ Medicine, Chicago, IL
}

\begin{abstract}
"Personalized" or precision medicine is a contemporary term applied to the oldest stopgap style of medical practice, reserved for when no one set of rules intended for the masses can be applied to the individual. This tailored approach describes the adjuvant treatment strategy for stage II colon cancer patients. Unlike stage III colon cancer patients, the role of adjuvant chemotherapy for stage II patients is uncertain. Overall, $25-40 \%$ of patients diagnosed with adenocarcinoma of the colon will have stage II disease, which portends a relatively good prognosis, with 5 -year overall survival rates ranging from 72 to $85 \%$. Conversely, up to $25 \%$ of stage II colon cancer patients will develop recurrent disease, and most of these patients will die as a result of their recurrence. With treatment en masse, routine administration of FOLFOX-based adjuvant chemotherapy to stage II colon cancer patients does not substantially improve overall survival compared with single-agent 5-fluorouracil. ${ }^{1}$ In an effort to better identify and treat stage II cancer patients with a high risk of recurrence, dozens of investigations have been published over the decades, but finding reliable prognostic factors has proven elusive.

Current guidelines advise against routine administration of adjuvant chemotherapy for stage II patients, except for
\end{abstract}

This is an editorial to the article available at doi: $10.1016 / \mathrm{S} 0016-$ 5085(13)62166-6.

(C) Society of Surgical Oncology 2014

First Received: 26 February 2014;

Published Online: 14 March 2014

M. F. McGee, MD, FACS

e-mail: mmcgee@nmff.org those with high-risk features such as poorly differentiated tumors, T4 disease (stage IIB/IIC), lymphovascular or perineural invasion, obstructing or perforated tumors, or threatened or positive margins and those with inadequate lymph node sampling. ${ }^{2}$ Although these clinical high-risk features empirically seem to place stage II patients at an increased risk of recurrence, there is scant evidence that adjuvant chemotherapy improves survival for such patients. $^{3,4}$ This paucity of data makes risk stratification difficult, leaving physicians to individualize treatment for stage II patients; this can result in wide practice variations. ${ }^{5,6}$

The heterogeneity of stage II patients has led countless investigators over the past decade on a hunt for the holy grail-identifying select stage II "high-risk" patients who would benefit from chemotherapy. Lymphovascular invasion, inadequate lymph node sampling, tumor differentiation, tumor budding, preoperative carcinoembryonic antigen levels, threatened (or close) resection margins, tumor immunology, biomarkers, and, more recently, genetic signature profiling have been carefully scrutinized in an attempt to identify a niche group of stage II patients who would benefit from adjuvant chemotherapy. ${ }^{7}$ Unfortunately, many of the data for these select patients come largely from subgroup, post hoc, and small series analyses that have been scrutinized for being underpowered. In the quest to identify stage II colon cancer patients who would benefit from adjuvant chemotherapy, it has been easier to identify those who should not receive chemotherapy than to identify patients who should.

One group of stage II patients that generally does not benefit from routine administration of FOLFOX-based adjuvant chemotherapy is those with high levels of microsatellite instability (MSI-H) associated with an 
identified mismatch repair (MMR) gene defect, who generally have a favorable prognosis even without adjuvant chemotherapy. ${ }^{8,9}$ An estimated $15 \%$ of colon cancers arise from defective DNA MMR genes, which are more frequently seen in younger patients with a predominance of right-sided cancers. Of these, approximately $13 \%$ are sporadic colon cancers, and the remainder represents those with germline mutations (hereditary nonpolyposis colorectal cancer). There are data that note improved survival after adjuvant chemotherapy, including oxaliplatin, for those MSI patients with cancers of germline origin but not those with sporadic MSI tumors. ${ }^{10,11}$ Recent analyses have shown that patients with the best prognosis have MSI-H and BRAF wild-type tumors. Whereas those with MSI-H and BRAF mutated tumors also have a good prognosis, they have a shorter survival time after recurrence compared with those with wild-type BRAF tumors. ${ }^{12}$

An estimated $20-25 \%$ of right-sided colon cancers are MSI-high, in contradistinction to left-sided colon cancers, which rarely express MMR gene defects. The differential of MSI status based on tumor location could theoretically divide stage II colon cancer patients into a group of left-sided MSI-stable patients who may benefit more from adjuvant chemotherapy versus a group of right-sided MSI-high patients who may not. An analysis from a large adjuvant stage III colon carcinoma clinical trial (FOLFOX \pm cetuximab) demonstrated that those individuals with an MMR defect (MSI-H) versus microsatellite stable tumors in the proximal colon have a better disease-free survival; however, those with an MMR defect versus proficient MMR tumors in the distal colon had a worse prognosis. Those with proficient MMR tumors in the proximal versus distal colon had a worse disease-free survival than those with defective MMR N2 versus $\mathrm{N} 1$ disease. $^{13}$

Leveraging the differential between MSI rates by tumor locations, Weiss et al. explore the relationship of tumor location (as a surrogate of MSI status), adjuvant chemotherapy use, and 5-year survival for stage II colon cancer in this issue of the Annals of Surgical Oncology. By using a retrospective review of a Medicare-linked Surveillance, Epidemiology, and End Results database from 1992 to 2005, overall survival after surgery and adjuvant chemotherapy was compared between right- and left-sided cancers for more than 4,500 stage II colon cancer patients. The authors report that there was no difference in survival between left- and right-sided colon cancer for stage II colon cancer patients receiving adjuvant chemotherapy. Weiss et al. conclude that tumor location does not influence response to adjuvant chemotherapy for stage II cancers. Unfortunately, the hypothesized relationship between tumor location, MSI status, and response to chemotherapy remains unsubstantiated and cannot be used as a factor to subselect stage II patients who would benefit from adjuvant chemotherapy.
As with most large databases, the large number of patient records is often obtained at the expense of data resolution and granularity, and the current study is no exception. Most notably, MSI status was not known for patients, and tumor location was used as a surrogate. For the authors' premise to work, one needs to assume that significantly more right-sided tumors are MSI-high compared with left-sided tumors, but this premise is assumed and not proven for the study group. Additionally, as the authors point out, chemotherapy status for the study population was based on administrative Medicare claims. This limitation means that only the presence of adjuvant chemotherapy was reported, whereas the duration, completion rates, and used chemotherapy agents were not tracked. Finally, the Medicare database is limited to patients aged 66 years and older. If one assumes that MSI-high tumors are more likely to be present in younger patients (e.g., Bethesda criteria), then such patients are practically excluded from the analysis, which diminishes the broader applicability of the study. On the basis of the data provided, the authors can state that for patients 66 years of age and older, tumor location alone does not influence the survival benefits of adjuvant chemotherapy for stage II colon cancer. It may be dubious to extrapolate the study findings to stage II patients who are 65 and younger, who may be more likely to have right-sided MSI-high tumors than the older population Weiss et al. describe.

Nonetheless, patients and clinicians alike are ravenous for data that can better identify which stage II patients will benefit from adjuvant chemotherapy, and the current study adds value to our understanding of this nuanced group. Weiss et al. make a noble attempt to guide the oncologic community but, like many other investigators, leave physicians clamoring for better means of stratifying high-risk stage II patients. The compendium of equivocal findings for several studies has clinicians mired in equipoise when considering adjuvant treatment for stage II patients. Not everyone needs a tailored suit: some people find a perfect fit right off the rack. Unfortunately, for stage II colon cancer patients, adjuvant chemotherapy remains a heavily tailored process for most patients and physicians.

\section{REFERENCES}

1. Andre T, Boni C, Navarro M, et al. Improved overall survival with oxaliplatin, fluorouracil, and leucovorin as adjuvant treatment in stage II or III colon cancer in the MOSAIC trial. $J$ Clin Oncol. 2009;27:3109-16.

2. Benson AB III, Bekaii-Saab T, Chan E, et al. Localized colon cancer, version 3.2013: featured updates to the NCCN guidelines. J Natl Compr Cancer Netw. 2013;11:519-28.

3. Peng SL, Thomas M, Ruszkiewicz A, Hunter A, Lawrence M, Moore J. Conventional adverse features do not predict response to adjuvant chemotherapy in stage II colon cancer. ANZ J Surg. 2013. doi:10.1111/ans.12444. 
4. O'Connor ES, Greenblatt DY, LoConte NK, et al. Adjuvant chemotherapy for stage II colon cancer with poor prognostic features. J Clin Oncol. 2011;29:3381-8.

5. Chagpar R, Xing Y, Chiang YJ, et al. Adherence to stage-specific treatment guidelines for patients with colon cancer. J Clin Oncol. 2012;30:972-9.

6. Earle CC, Weiser MR, Ter Veer A, et al. Effect of lymph node retrieval rates on the utilization of adjuvant chemotherapy in stage II colon cancer. J Surg Oncol. 2009;100:525-8.

7. Yothers G, O'Connell MJ, Lee M, et al. Validation of the 12-gene colon cancer recurrence score in NSABP C-07 as a predictor of recurrence in patients with stage II and III colon cancer treated with fluorouracil and leucovorin (FU/LV) and FU/LV plus oxaliplatin. J Clin Oncol. 2013;31:4512-9.

8. Sargent DJ, Marsoni S, Monges G, et al. Defective mismatch repair as a predictive marker for lack of efficacy of fluorouracilbased adjuvant therapy in colon cancer. J Clin Oncol. 2010;28:3219-26.
9. Ribic CM, Sargent DJ, Moore MJ, et al. Tumor microsatelliteinstability status as a predictor of benefit from fluorouracil-based adjuvant chemotherapy for colon cancer. $N$ Engl $J$ Med. 2003;349:247-57.

10. Damia G, D'Incalci M. Genetic instability influences drug response in cancer cells. Curr Drug Targets. 2010;11:1317-24.

11. Buecher B, Cacheux W, Rouleau E, Dieumegard B, Mitry E, Lievre A. Role of microsatellite instability in the management of colorectal cancers. Dig Liver Dis. 2013;45:441-9.

12. Gavin PG, Colangelo LH, Fumagalli D, et al. Mutation profiling and microsatellite instability in stage II and III colon cancer: an assessment of their prognostic and oxaliplatin predictive value. Clin Cancer Res. 2012;18:6531-41.

13. Sinicrope FA, Mahoney MR, Smyrk TC, et al. Prognostic impact of deficient DNA mismatch repair in patients with stage III colon cancer from a randomized trial of FOLFOX-based adjuvant chemotherapy. J Clin Oncol. 2013;31:3664-72. 